\title{
VISUAL ANALYTICS WEB PLATFORM FOR DETECTING HIGH WIND ENERGY POTENTIAL IN URBAN ENVIRONMENTS BY EMPLOYING OGC STANDARDS
}

\author{
Athanasios Koukofikis*, Volker Coors \\ University of Applied Sciences Stuttgart \\ Schellingstraße 24 \\ 70174 Stuttgart, Germany \\ (athanasios.koukofikis, volker.coors)@hft-stuttgart.de
}

Commission VI, WG VI/4

KEY WORDS: Visual analytics, CFD, Wind energy, OGC, 3DPS, Web Visualization, WebGL

\begin{abstract}
:
We propose a server-client web architecture identifying areas with high wind energy potential by employing 3D technologies and OGC standards. The assessment of a whole city or sub-regions will be supported by integrating Computational Fluid Dynamics (CFD) with historical wind sensor readings. The results, in 3D space, of such analysis could be used for locating installation points of small-scale vertical axis wind turbines in an urban area.
\end{abstract}

\section{INTRODUCTION}

The urban fabric could be a prosperous environment for wind energy harnessing. Several studies were conducted by researchers in order to profile and assess the energy potential in city blocks or entire cities. Simulation processes, specifically Computational Fluid Dynamics (CFD), play a fundamental role in numerically estimating wind velocity and direction in a segregated/sampled 3D space. Although in an urban context a CDF simulation could have a high error tolerance, mainly for performance improvement, the calculation of a dynamic velocity field in the course of a year is greatly demanding. Moreover, the toolsets and methodologies for performing such simulations are not easily accessible for non-experts with interest of assessing the energy yield of a small scale wind turbine installation on a building. The evolution in data-driven web based visualization allows for interactive exploration and information extraction in a wide audience. The latest revision of the HTML language enables hypertext to render interactive/high performance 2D/3D graphics utilizing the WebGl API without the need for a plugin.

\section{STATE OF THE ART}

The research community so far utilized various methods to perform wind energy estimation in urban areas. Authors (Wang et al., 2018) compared and analyzed seven different urban tissues using CFD simulations in the area of Beijing. The numeric results of the simulations were correlated with urban morphology parameters such as floor area ratio, plot ratio, average building height, standard deviation of the building heights, mean building volume, relative rugosity and porosity. The results showed that urban areas with high porosity, high average building height and low floor area ratio can yield more energy from the wind. In the wider area of Copenhagen (Beller, 2011) performed a wind seasonality analysis utilizing wind direction and wind velocity readings on the roof of H.C. Ørsted Institute building in a period over three years. The annual energy

\footnotetext{
* Corresponding author
}

production was estimated along with an evaluation of the performance of three small-scale wind turbines virtually exposed to all wind directions found at the roof of the building of H.C. Ørsted Institute. In Helsinki's Kalasatama area, throughout the digital twins project (Suomisto et al., 2019), a high accuracy DSM model was generated for visualization purposes and a semantic city model based on CityGML. The CityGML model was used in a CFD simulation in order to investigate the wind impact on the micro climate, comfort and safety of the streets and pedestrian areas. Authors (Chtibi, Touzani, 2019) presented a study of wind energy potential in the area of Morocco based on reports by the Moroccan Ministry of Energy, of the Mines, of the Water and Environment. Authors (Wilkinson et al., 2014) demonstrated a new approximation approach of computational fluid dynamics in high rise buildings with complex wind interference.

In the web visualization domain numerous studies realized the emerging technologies utilizing declarative and imperative graphics rendering pipelines. Authors (Gaillard et al., 2015) implemented a view-dependent 3D city client-server architecture based on CityGML as a source information model. 3DImpact is an interactive web application developed by (Patterson, 2016) that accesses World Health Organization data, in conjunction with spatial information, to visualize spatio-temporal phenomena using a virtual 3D globe based on Google Earth. Authors (Prieto, Izkara, 2012) produced web visualizations of 3D city models on mobile devices using X3DOM, driven by the use of CityGML as the reference data model. Authors (Klimenko et al., 2018) implemented in the virtual reality realm a web application that visualizes in 3D space the similarity of scientific papers. Authors (Friston et al., 2019) introduced a novel method for rectangular selection of components in large 3D models on the web. This technique can be adopted by embedded systems running on WebGL. Authors (Döllner et al., 2012) presented a server-side rendering pipeline of 3D scenery including clientside reconstruction based on geometry buffers. 


\section{PROBLEM STATEMENT}

3D visualization is the fundament to the establishment of a modern information system in an urban space. An interactive visualization, based on 3D city modeling, can extract meaningful information in the field of wind energy. Identifying locations in an urban environment where a small scale wind turbine could yield an adequate amount of energy in the course of a year is a four dimensional problem. Three dimensions are used for the spatial component of the location, i.e. the $\mathrm{X}, \mathrm{Y}, \mathrm{Z}$ coordinates in an arbitrary coordinate system where wind speed reaches above a required threshold. The fourth dimension is the time component, i.e., the duration of the required wind speed during a year. State of the art approaches utilize wind speed and direction readings to profile an urban area and to investigate its suitability or use numeric solutions via CDF simulations in order to generate a dynamic or static wind velocity field. The wind potential assessment of a city model could be used in tandem with other independent city models and utilize a similarity factor of the 3D city model meshes, i.e., the Hausdorff distance (Hausdorff, 1965), in order to assess them skipping the simulation step. Such approach would require similar terrain surface model and the same wind profile (wind directions, wind speeds) for the additional city models.

Performing a dynamic CDF simulation for a whole year would need an enormous computer processing power and would produce a gigantic result in terms of computer storage space. Alternatively, averaging the wind velocity readings for coarser temporal resolutions, i.e., monthly or yearly would introduce inaccuracies in the CFD results. A week with average wind velocity of $7 \mathrm{~m} / \mathrm{s}$ and three weeks of average wind velocity of $1-2 \mathrm{~m} / \mathrm{s}$ would give a monthly average less than $3 \mathrm{~m} / \mathrm{s}$, which is not a recommended wind velocity for a small scale wind turbine. Therefore, a week ( $\operatorname{avg} 7 \mathrm{~m} / \mathrm{s}$ ) with high potential of wind energy is not utilized. On the other side a static CDF simulation would be insufficient as it is missing the time component. Moreover, the state of the art solutions require high expertise in the area of wind energy and CFD simulations. A web based client-server architecture can be used to assess the wind energy potential in an urban environment by integrating static CFD simulations, wind historical data, Web 3D technologies and OGC Standards which would allow researchers, entrepreneurs and civilians to estimate the yearly yield of such investment. A spatio-temporal query in the form of "find locations where the wind velocity is higher than $6 \mathrm{~m} / \mathrm{s}$ for more than 5 hours a day for more than 200 days in a year" is expected to be resolved by such a solution. The results of such query will indicate locations where a small scale vertical axis wind turbine can be installed.

\section{METHODOLOGY}

Historical wind data are provided from the Office of Environmental Protection, Section of Urban Climatology of city of Stuttgart (Stadtklima-stuttgart.de, 2020). The data are available in various temporal resolutions (yearly -30 minutes). The weather station is located 10 meters above the ground mounted on a building in the Stockach area, center of Stuttgart. A statistical analysis is conducted in order to identify the two most prominent wind directions during a whole year. Then for each wind direction the two wind speeds with the highest frequency are chosen. For each combination of wind direction/wind speed a static CDF simulation is performed (short time span) with boundary conditions the relevant wind direction and wind speed at 10 meters above ground. The resulting 3D vector fields will be stored in a relational database and will be accessed by the application layer. Assuming that the city model geometry has not been altered by the time of the CFD simulations were conducted, when an event in the historical wind data is found with low tolerance in the wind direction and speed, we assume the same boundary conditions as the CFD simulations and the same numerical results (Figure 1).

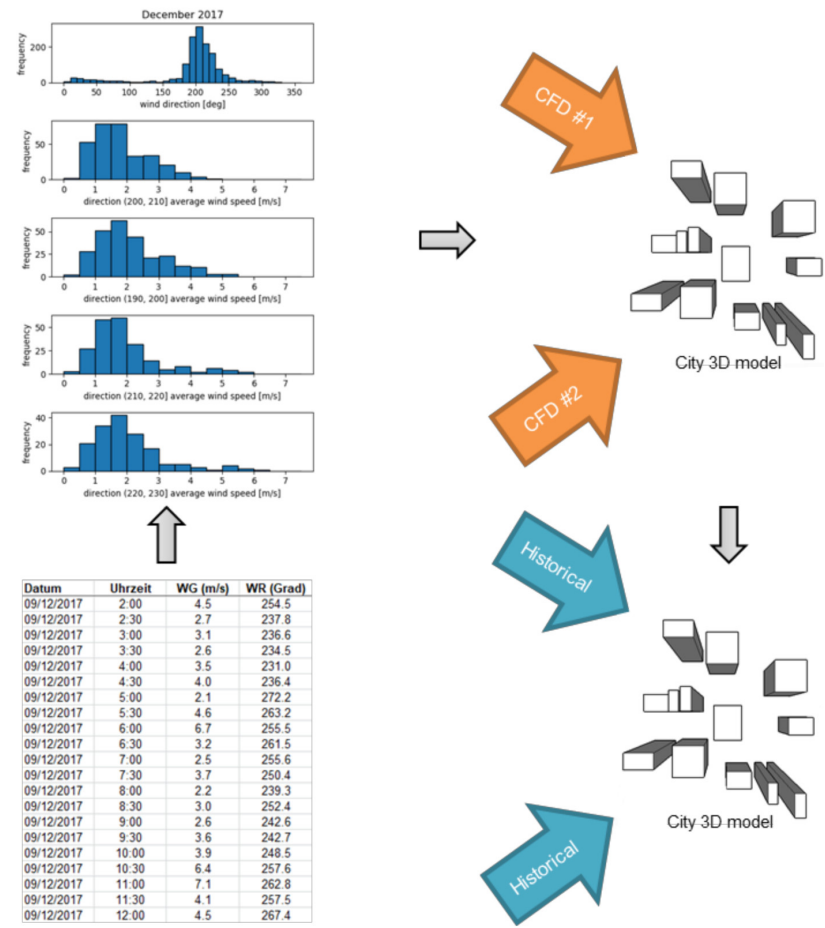

Figure 1. Calculation of boundary conditions for performing static CFD simulations

The application layer will generate a feature 3D grid which will cover the whole city model until a specified relative height above ground. Then, a voting scheme will aggregate events in the historical wind data that qualify the spatial, temporal and attributive parameters given by the user (Figure 2).
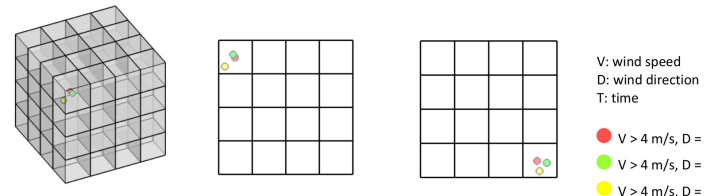

$V>4 \mathrm{~m} / \mathrm{s}, \mathrm{D}=270 \mathrm{deg}, \mathrm{T}=15: 00$ $V>4 \mathrm{~m} / \mathrm{s}, \mathrm{D}=250 \mathrm{deg}, \mathrm{T}=15: 30$ side view

top view $V>4 \mathrm{~m} / \mathrm{s}, \mathrm{D}=270 \mathrm{deg}, \mathrm{T}=16.00$

Figure 2. A 3D feature grid with three historical events in a cell. The dots represent the locations of the velocity vectors. The red and yellow vector are results of the same CDF simulation since they are produced from the same wind direction (assuming the same wind speed in the historical data)

The access of the 3D assets will be abstracted via an OGC's web interface, namely the 3D Portrayal Service. The web client will be based on a custom tag library, similar to the leading React.js library, which extends the semantics of an HTML document. This allows the freedom of modularity and versatility by a developer perspective. The overall architecture (Figure 3 ) would allow users, independent of level of expertise in the wind energy field, to locate areas in a $3 \mathrm{D}$ environment with high wind 
energy potential. 3DPS would play the important role of the access web interface of 3D meshes and wind field information.

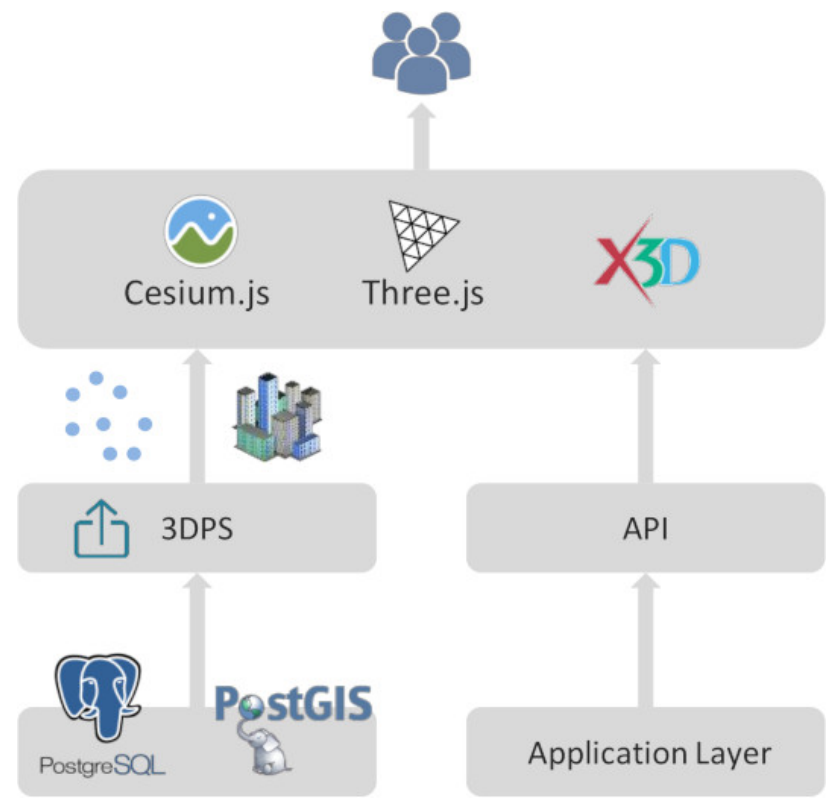

Figure 3. Overall architecture of the application

On the client side the user will be able to navigate and visually analyze the simulation data utilizing a multi-layer scheme (Figure 4). The web client will be based on Three.js, a powerful 3D engine bringing computer vision to the browser, and Riots.js, a library which shares commonalities with React.js, a popular client side framework/library, offering custom HTML tags which are the building blocks of the user interface. The user interface will be composed by a Single Page Application. The layout will be separated into two main sections: The 3D Engine viewer on the right side and the side-bar on the left side of the screen. The 3D viewer will render the $3 \mathrm{D}$ static assets, i.e. buildings, terrain and dynamically generated query results or CDF attributes. Additionally, the user will be able to interact with the 3D viewer, via defining clipping planes, in order improve the visualization of results in specific city volumes. The side-bar will be a scrollable generic area of graphical user interface to control the workflow. Various user interface controls will be logically grouped and wrapped in collapsable graphical elements in order to save space on the side-bar. A group of user interface controls will define the 3D spatial domain as an offset of a tight bounding volume of the city model in order to exclude CFD data (e.g. wind velocity 100 meters above ground when the highest building is 30 meters) and improve performance. Every visualization result will be stored as a separate layer and grouped in a collapsable graphical element in the side-bar. Every layer will have controls for hiding, editing and deletion. Editing of a layer will take advantage of the client-side routing of Riot.js and rerender a different layout without accessing the server (Figure 5). The user will formulate a query giving as input the desired wind speed threshold, the duration of continuous wind speed in daily basis and the number of days in a year. A feature layer will then be generated by utilizing the wind historical data and the static CFD wind fields according to the prevailing wind directions and wind speeds for these directions. When a similar (to a certain extent) wind direction and wind speed is found in the historical data, the corresponding static CFD simulation will be used and the time resolution of the historical wind data will be accumulated.
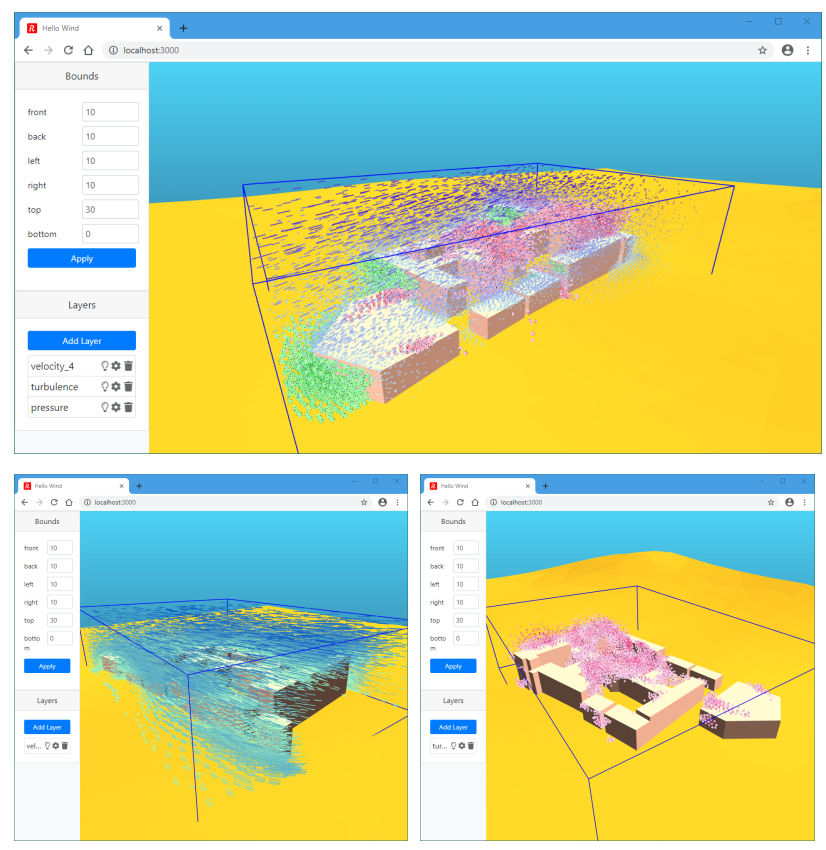

Figure 4. A prototype web client for identifying high wind energy potential locations using the Three.js runtime

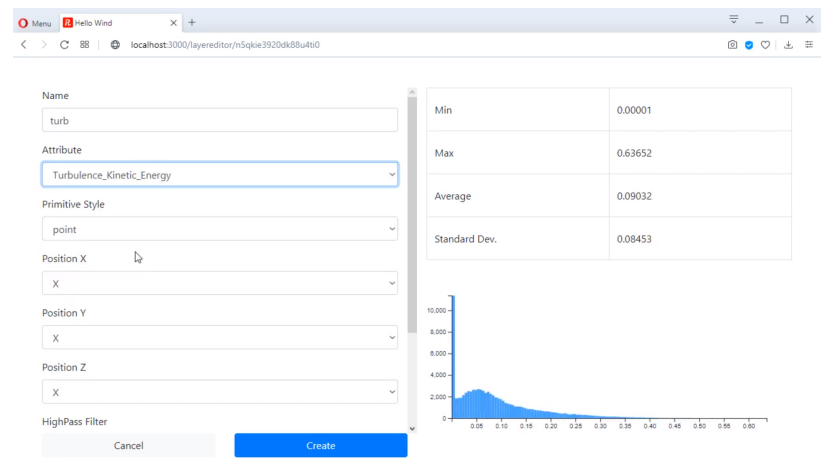

Figure 5. Creation/editing of a layer will re-render a new layout without a server request

\section{INTEROPERABLE VISUALIZATION USING THE 3D PORTRAYAL SERVICE (3DPS)}

The 3D Portrayal Service (Hagedorn et al., 2017) is a OGC Standard that abstracts the access of 3D geospatial datasets in various client platforms via the web for visualization purposes. The 3D Portrayal Service specifies three methods to access information: GetCapabilities, AbstractGetPortrayal and AbstractGetFeatureInfo. A GetCapabilities request returns information about the available request methods, the extents of the data, data layers (buildings, vegetation etc.), layer styles and streaming formats supported. The retrieval of a scene is implemented by the AbstractGetPortrayal operator. The GetScene method is used for client side rendering and the GetView method for server side rendering. The AbstractGetFeatureInfo implementations are used for requesting metadata of scene features. The supported methods are GetFeatureInfoByObjectId, GetFeatureInfoByPosition and GetFeatureInfoByRay. The 3D Portrayal Service specification does not indicate a content delivery format. Among several payload formats OGC community standards which expose a bounding volume hierarchy, namely, 3D-Tiles and I3S, can be served by a 3DPS GetScene imple- 
mentation. 3D-Tiles is an open specification for sharing, visualizing, fusing, and interacting with massive heterogenous 3D geospatial content across desktop, web, and mobile applications (Cozzi et al., 2018). 3D-Tiles allows data providers and app developers to make massive and complex 3D information more accessible, interoperable, and useful across all kinds of tools and applications. I3S (Indexed 3D Scene Layer) is a technology for rapidly streaming and distributing large volumes of $3 \mathrm{D}$ content across enterprise systems that may consist of server components, cloud hosted components, and desktop, web and mobile clients (ESRI, 2019). The specification is realized by various profiles that describe the behavior of a I3S layer. The spatial extent of a I3S layer is split into bounding volumes, described as nodes, and organized into a hierarchical data structure that allows a client to quickly discover and request which geodata should be rendered.

\section{CONCLUSIONS}

This paper introduces a client-server architecture for locating high wind energy potential inside the urban environment by integrating simulation outcomes, historical wind data, web technologies and OGC standards. The preliminary results (Figure 4, Figure 5) show the visualization of static CFD attributes in a small region in the center of Stuttgart, Germany, with the temporal component of the analysis being absent. In the future, the addition of the temporal component and realization of the spatio-temporal query will follow the implementation of OGC's 3D Portrayal Service which is proven to be an effective interface for 3D content delivery.

\section{ACKNOWLEDGEMENTS}

The authors would like to thank Ordnance Survey GB (www.ordnancesurvey.co.uk) and 1Spatial (www.1spatial.com/) for sponsoring the publication of this paper

\section{REFERENCES}

Beller, C., 2011. Urban Wind Energy. PhD thesis.

Chtibi, A., Touzani, A., 2019. Actual wind energy use situation worldwide and in Morocco: Case of small vertical axis wind turbine market offers. Proceedings of the 4th International Conference on Big Data and Internet of Things, 1-7.

Cozzi, P., Lilley, S., Getz, G., 2018. 3D Tiles Specification. https://github.com/CesiumGS/3d-tiles/tree/master /specification.

Döllner, J., Hagedorn, B., Klimke, J., 2012. Server-Based Rendering of Large 3D Scenes for Mobile Devices Using G-Buffer Cube Maps. The 17th International Conference on $3 D$ Web Technology, Association for Computing Machinery, New York, NY, USA, 97-100.

ESRI, 2019. I3S Specification. https://github.com/Esri/i3sspec.

Friston, S., Doboš, J., Wong, C., Fan, C., Montero, S., Steed, A., 2019. Rectangular Selection of Components in Large 3D Models on the Web. The 24th International Conference on 3D Web Technology, Web3D '19, Association for Computing Machinery, New York, NY, USA, 1-9.
Gaillard, J., Vienne, A., Baume, R., Pedrinis, F., Peytavie, A., Gesquière, G., 2015. Urban Data Visualisation in a Web Browser. Proceedings of the 20th International Conference on $3 D$ Web Technology, Web3D '15, Association for Computing Machinery, New York, NY, USA, 81-88.

Hagedorn, B., Thum, S., Reitz, T., Coors, V., Gutbell, R., 2017. 3D PORTRAYAL SERVICE version 1.0. $\mathrm{http} / / /$ docs.opengeospatial.org/is/15-001r4/15-001r4.html.

Hausdorff, F., 1965. Grundzuege der Mengenlehre. Chelsea.

Klimenko, S., Charnine, M., Zolotarev, O., Merkureva, N., Khakimova, A., 2018. Semantic Approach to Visualization of Research Front of Scientific Papers Using Web-Based 3D Graphic. Proceedings of the 23rd International ACM Conference on 3D Web Technology, Web3D '18, Association for Computing Machinery, New York, NY, USA.

Patterson, D., 2016. Interactive 3D Web Applications for Visualization of World Health Organization Data. Proceedings of the Australasian Computer Science Week Multiconference, ACSW '16, Association for Computing Machinery, New York, NY, USA.

Prieto, I. n., Izkara, J. L., 2012. Visualization of 3D City Models on Mobile Devices. Proceedings of the 17th International Conference on 3D Web Technology, Web3D '12, Association for Computing Machinery, New York, NY, USA, 101-104.

Stadtklima-stuttgart.de, 2020. Download von Messdaten der Station S-Mitte, Schwabenzentrum. https://www.stadtklimastuttgart.de/index.php?luft_messdaten_download.

Suomisto, J., Airaksinen, E., Bergstrom, M., Heinonnen, H., Lahti, K., Kaisla, K., 2019. The Kalasatama Digital Twins Project. Technical report, Helsinki 3D+.

Wang, B., Sun, S., Duan, M., 2018. Wind potential evaluation with urban morphology - A case study in Beijing. Energy Procedia, 153, 62 - 67. 5th International Conference on Energy and Environment Research, ICEER 2018, 23-27 July 2018, Prague, Czech Republic.

Wilkinson, S., Bradbury, G., Hanna, S., 2014. Approximating Urban Wind Interference. Proceedings of the Symposium on Simulation for Architecture \& Urban Design, SimAUD '14, Society for Computer Simulation International, San Diego, CA, USA.

Revised January 2020 